\title{
Minimum heat dissipation in measurement-based quantum computation
}

\author{
Tomoyuki Morimat* \\ ASRLD Unit, Gunma University, 1-5-1 Tenjin-cho Kiryu-shi Gunma-ken, 376-0052, Japan
}

(Dated: February 13, 2018)

\begin{abstract}
We show that at least $2 k T \ln 2$ of heat dissipation per qubit occurs in measurement-based quantum computation according to Landauer's principle. This result is derived by using only the fundamental fact that quantum physics respects the no-signaling principle.
\end{abstract}

The search for faster, smaller, and more economical computers is the central research subject in today's ever growing digital society. Heat generation (or equivalently energy consumption) during computation is one of the huge obstacles to above goals, and in fact it has been a long standing research topic in the interdisciplinary field between information and thermodynamics [1 10]. In 1961, Landauer showed in his seminal paper [1] that an irreversible process, such as an erasure of data in a memory, inevitably causes a minimum amount of heat dissipation. More precisely, so called Landauer's principle says that an erasure of a single bit of information in a memory causes at least $d S=k \ln 2$ of entropy generation (or, in other words, $\delta Q=k T \ln 2$ of heat dissipation or energy consumption, since $\delta Q=d S T$ ), where $k$ is the Boltzmann constant and $T$ is the temperature of the environment. Landauer's principle has been used not only to exorcise Maxwell's demon in thermodynamics [9] but also to establish fundamental limits of heat generation and energy consumption in irreversible computation.

Although heat dissipation in quantum computers has not yet been fully studied, it will be a crucial problem as well in a near future when we can access small realistic quantum computers. Due to the intrinsic decoherence nature of quantum computers, the importance of studying minimum heat dissipation in quantum computation will be arguably more emphasized than that in classical computation. The purpose of this paper is to study minimum heat dissipation in measurement-based quantum computation (MBQC) [1]. We show that at least $2 k T \ln 2$ of heat dissipation per qubit occurs in MBQC according to Landauer's principle (or an inequality due to Sagawa and Ueda [10]). Interestingly, this result is independent from any specific physical implementation of MBQC, and in fact derived by using only the fundamental fact that quantum physics respects the no-signaling principle 12]. We will also see that MBQC with the cluster state [1] already achieves this minimum heat dissipation limit, and therefore our result is an achievable lower bound.

Our result also exorcises Maxwell's demon in MBQC. Since MBQC uses adaptive measurements, it is a kind of a feed-back controlled system with a demon. However, no result was obtained about the study of MBQC from the view point of the feed-back control. The output of MBQC is a (classical or quantum) element ex- tracted from many possibilities, and therefore MBQC is an entropy decreasing process. However, the entire system (i.e., the resource state of $\mathrm{MBQC}$, the measuring apparatus, the classical computer necessary for the feedforwarding, and the environment) is a closed system, and therefore the entropy decrease must be compensated by an entropy increase of another degrees of freedom; otherwise the second law of thermodynamics is violated. Such a Maxwell's demon problem in MBQC is solved by our result: an entropy increase necessarily occurs in MBQC (because of the classical memory requirement for the demon as we will see later), and it compensates the entropy decrease caused by the demon's computation.

No-signaling principle.- Let us quickly review the nosignaling principle on which our result is based. Nosignaling principle is one of the most fundamental principles in physics, and quantum theory also respects it [12]. As is shown in Fig. 1 let us assume that Alice and Bob share a physical system, which might be classical, quantum, or even super-quantum. Alice chooses her measurement parameter $x$ (such as the measurement angle of a spin), and performs measurement on her part. She obtains the result $a$. Bob also chooses his measurement parameter $y$, and performs measurement on his part. He obtains the result $b$. The no-signaling principle (from Alice to Bob) is defined by

$$
P(b \mid x, y)=P\left(b \mid x^{\prime}, y\right)
$$

for all $b, x, x^{\prime}$, and $y$, where $P(\alpha \mid \beta)$ is the conditional probability distribution of $\alpha$ given $\beta$. Equation (11) means that the change of Alice's measurement parameter does not affect the probability distribution of Bob's measurement result. In other words, the shared system cannot transmit any message from Alice to Bob. Interestingly, the no-signaling principle is more fundamental than quantum theory in the sense that there is a theory which is more non-local than quantum theory, but respects the no-signaling principle [12].

General MBQC.- Measurement-based quantum computation is a new model of quantum computation introduced by Raussendorf and Briegel [11]. In this model, universal quantum computation can be done with only the preparation of a highly-entangled quantum manybody state so called a resource state, and adaptive local measurements on each qubit of the resource state. Here, adaptive means that a measurement basis depends 


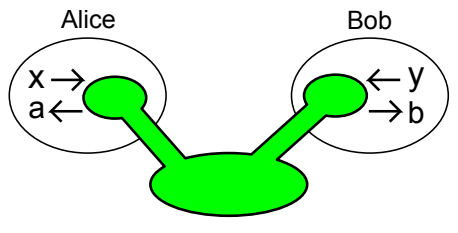

FIG. 1: The no-signaling principle.

on the previous measurement results. Hence, in addition to the resource state, which is a quantum system, we need a classical computer to process measurement results (Fig. 2).

The computational power of MBQC is equivalent to the traditional circuit model of quantum computation, but the clear separation between the quantum phase (preparation of the resource state) and the classical phase (local adaptive measurements) has inspired many new results which would not be obtained if we stick to the circuit model. For example, new resource states for MBQC which are closely connected with condensed matter physics have been proposed [13 23]. Furthermore, a relation between $\mathrm{MBQC}$ and partition functions of classical spin models were pointed out [24 26]. These discoveries have established a new bridge between quantum information and condensed matter physics. MBQC has also offered a new framework for fault-tolerant quantum computing which achieves high threshold [27 32]. The quantum-classical separation in MBQC has enabled us to clarify several relations between "quantumness" of a resource state and quantum computational power of MBQC on it 33 38]. New protocols of secure cloud quantum computing, so called blind quantum computing, were also developed by using MBQC [39 53].

In the most general framework of MBQC, we first prepare the resource state, $\sigma$, of $N=n m$ qubits as is shown in Fig. 3. Qubits are allocated on sites of the $n \times m$ twodimensional square lattice, and MBQC simulates a quantum circuit with the register size $n$ and the gate depth $m-1$. (More generally, we can consider more general graph structure and qudits, but generalization is straightforward. For simplicity, we consider two-dimensional square qubit system.) Be careful that $\sigma$ is not necessarily the cluster state [11]. We do not assume any specific resource state as $\sigma$. Measurements on all qubits in $j$ th layer of $\sigma$ implement the $n$-qubit unitary gate $U_{j}$. The initial state of the computation is the $n$-qubit state $\rho_{i n}$, and it is encoded in the first layer of $\sigma$. Let $C_{r}$ be the set of all qubits in the first $r$ layers of $\sigma$ (Fig. 3). We also define $O_{r}$, which is the set of all qubits in the last $m-r$ layers of $\sigma$ (Fig. 3).

According to the standard theory of quantum measurement 54], a measurement process on $C_{r}$ is described as follows. First, an correlation between the measurement apparatus and the system $\sigma$ to be measured is created:

$$
\sum_{j=1}^{c} p_{r}^{j}|j\rangle\langle j| \otimes \mathcal{E}_{r}^{j}(\sigma),
$$

where $\mathcal{E}_{r}^{j}$ is a CPTP map, and $p_{r}^{j}$ is a probability. (Offdiagonal terms are omitted here for simplicity.) Next, the projection measurement $\{|j\rangle\langle j|\}_{j=1}^{c}$ is performed on the apparatus, which leads to the post-measurement state $\mathcal{E}_{r}^{j}(\sigma)$ with the probability $p_{r}^{j}$.

We require that

$$
\begin{aligned}
\rho_{\text {out }, r}^{j} & \equiv \operatorname{Tr}_{C_{r}}\left[\mathcal{E}_{r}^{j}(\sigma)\right] \\
& =B_{r}^{j}\left[\left(U_{r} \ldots U_{1} \rho_{\text {in }} U_{1}^{\dagger} \ldots U_{r}^{\dagger}\right) \otimes \eta_{r}^{j}\right] B_{r}^{j \dagger},
\end{aligned}
$$

where $\operatorname{Tr}_{C_{r}}$ is the partial trace over $C_{r}, B_{r}^{j}$ is an $(m-r) n$ qubit unitary operator, and $\eta_{r}^{j}$ is a state of $(m-r) n-n$ qubits. The reason why we require the form of $\rho_{\text {out }, r}^{j}$ in that way can be easily understood if we remember that $\rho_{\text {out }, r}^{j}$ must contain the complete information about $U_{r} \ldots U_{1} \rho_{i n} U_{1}^{\dagger} \ldots U_{r}^{\dagger}$. (If not, we cannot proceed MBQC with $O_{r}$ ). More precisely, it was shown in Ref. [55] that any invertible CPTP map can be written as an application of a unitary operator on the system plus ancilla. Therefore, the invertible CPTP map

$$
H_{n} \ni U_{r} \ldots U_{1} \rho_{\text {in }} U_{1}^{\dagger} \ldots U_{r}^{\dagger} \mapsto \rho_{\text {out }, r}^{j} \in H_{(m-r) n}
$$

has to be the form of Eq. (2), where $H_{d}$ is the $d$-qubit Hilbert space. For example, if $\sigma$ is the cluster state, $\eta_{r}^{j}$ is the $n \times(m-r-1)$ cluster state, and $B_{r}^{j}$ is the operation which applies $C Z$ gates on the border between $C_{r}$ and $O_{r}$, and random Pauli operators on $(r+1)$ th layer.

In particular, after measuring all qubits except for those in the last layer, the state of the last layer becomes

$$
\rho_{\text {out }, m-1}^{j}=B_{m-1}^{j} U \rho_{\text {in }} U B_{m-1}^{j \dagger}
$$

with probability $p_{m-1}^{j}$, where $U \equiv U_{m-1} U_{m-2} \ldots U_{2} U_{1}$. The operator $B_{m-1}^{j}$ is an unwanted operator, so called the byproduct operator, but we can correct it if we know $j$. In such an MBQC, we say that our desired unitary $U$ is implemented on $\rho_{i n}$ up to the byproduct $B_{m-1}^{j}$.

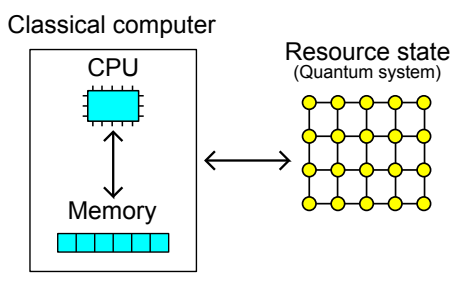

FIG. 2: Measurement results on the resource state are processed on a classical computer. 


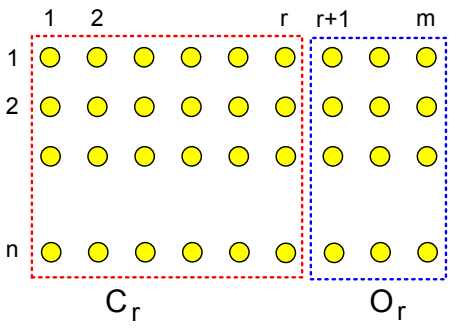

FIG. 3: The resource state $\sigma$ for MBQC.

Minimum heat dissipation in general MBQC.- Now let us show that in the above general MBQC, at least $2 k T \ln 2$ of heat dissipation per qubit is necessary. In order to see it, let us consider MBQC between two party, Alice and Bob as is shown in Fig. 4. The resource state $\sigma$ is shared between Alice and Bob. Alice possesses the subsystem $C_{r}$ and Bob does $O_{r}$. Alice performs MBQC on her part.

The state immediately before Alice performing the measurement on her apparatus is

$$
\sum_{j=1}^{c} p_{r}^{j}|j\rangle\langle j| \otimes \mathcal{E}_{r}^{j}(\sigma)
$$

If we trace out Alice's system, we obtain Bob's system,

$$
\rho_{o u t, r}^{B o b} \equiv \sum_{j=1}^{c} p_{r}^{j} \rho_{o u t, r}^{j}
$$

(a)

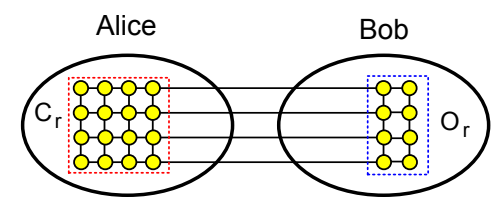

(b)

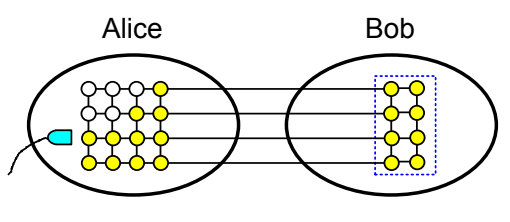

(c)

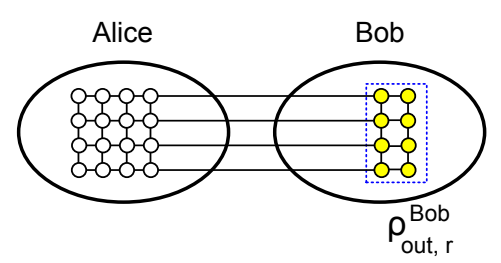

FIG. 4: Two party general MBQC between Alice and Bob.

Now we point out that $\rho_{\text {out }, r}^{\text {Bob }}$ must be completely independent from $U_{r} \ldots U_{1} \rho_{i n} U_{1}^{\dagger} \ldots U_{r}^{\dagger}$, since otherwise Bob can gain some information about $U_{r} \ldots U_{1} \rho_{i n} U_{1}^{\dagger} \ldots U_{r}^{\dagger}$ by measuring $\rho_{\text {out }, r}^{B o b}$. If Bob gains some information about
$U_{r} \ldots U_{1} \rho_{i n} U_{1}^{\dagger} \ldots U_{r}^{\dagger}$, it contradicts to the no-singling principle, because Alice can send some message to Bob by encoding her message into $U_{r} \ldots U_{1} \rho_{i n} U_{1}^{\dagger} \ldots U_{r}^{\dagger}$. In Refs. $[55-$ 57], it was shown that the entropy $H\left(p_{r}^{1}, \ldots, p_{r}^{c}\right)$ of $\left\{p_{r}^{j}\right\}_{j=1}^{c}$ must satisfy

$$
H\left(p_{r}^{1}, \ldots, p_{r}^{c}\right) \equiv-\sum_{j=1}^{c} p_{r}^{j} \log p_{r}^{j} \geq 2 n
$$

if the map

$$
H_{n} \ni \xi \mapsto \sum_{j=1}^{c} p_{r}^{j} B_{r}^{j}\left(\xi \otimes \eta_{r}^{j}\right) B_{r}^{j \dagger} \in H_{(m-r) n}
$$

works as the completely secure quantum one-time pad encryption for any $n$-qubit state $\xi$.

Therefore, Alice has to gain at least $2 n$ bit (or $2 n \ln 2$ nit in the natural base) of information when she measures her apparatus in Eq. (3). The acquisition of information is accompanied by the erase of it. According to Landauer's principle, this means that at least $2 n k T \ln 2$ of heat is dissipated when the data in the memory is erased. Therefore, we conclude that at least $2 k T \ln 2$ of heat dissipation per qubit occurs in MBQC.

Landauer's principle is not a mathematical theorem, but an "observation" derived from physically reasonable arguments. In order to obtain more precise statement, we have to specify the model. For example, if the erasure model is described by the thermalization of the memory interacting with a heat bath, Landauer's principle can be derived [8]. Recently, Sagawa and Ueda [10] introduced an inequality which generalizes Landauer's principle by assuming the following memory model: the state of the memory storing $j$ th result is given by the canonical state $\rho_{j, \text { can }}^{M}=\exp \left(-H_{j}^{M} / k T\right) / Z_{j}^{M}$, where $H_{j}^{M}$ is the Hamiltonian of the memory if it stores $j$ th result and $Z_{j}^{M} \equiv \operatorname{Tr}\left[\exp \left(-H_{j}^{M} / k T\right)\right]$. The state of the memory before erasure is given by $\sum_{j} p_{j} \rho_{j, \text { can }}^{M}$. In order to erase data in the memory, we couple the memory with the heat bath, $\rho_{\text {can }}^{B} \equiv \exp \left[-H^{B} / k T\right] / Z^{B}$, where $H^{B}$ is the Hamiltonian of the bath, and $Z^{B} \equiv \operatorname{Tr}\left[\exp \left(-H^{B} / k T\right)\right]$. The memory plus bath unitary time evolve to the final state $\rho^{M B}$. Under these assumptions, they derived

$$
W_{\text {eras }}+\Delta F^{M} \geq k T H,
$$

where

$$
\begin{aligned}
W_{\text {eras }} \equiv & \operatorname{Tr}\left(\rho^{M B}\left(H_{0}^{M}+H^{B}\right)\right) \\
& -\sum_{j} p_{j} \operatorname{Tr}\left(\rho_{j, \text { can }}^{M} H_{j}^{M}\right)-\operatorname{Tr}\left(\rho_{\text {can }}^{B} H^{B}\right)
\end{aligned}
$$

is the work required for the erasure of data in the memory, and $\Delta F^{M} \equiv k T \ln Z_{0}^{M}-\sum_{j} p_{j} k T \ln Z_{j}^{M}$ is the change of the free energy of the memory due to the erasure [58]. According to Eq. (4), we obtain $W_{\text {eras }}+$ 
$\Delta F^{M} \geq 2 n k T \ln 2$. In particular, if we consider the case when the memory's Hamiltonian does not depend on the stored data, i.e., $H_{j}^{M}=H_{0}^{M}$ for all $j$, we conclude that $W_{\text {eras }} \geq 2 n k T \ln 2$, which means that at least $2 k T \ln 2$ of work per qubit is necessary in MBQC.

Minimum heat dissipation in the cluster state $M B Q C$. - As an example, let us consider the minimum heat dissipation limit in the cluster state MBQC [11]. In the cluster state $\mathrm{MBQC}$, it is well known that measurement results for two previous layers must be kept in order to correct byproduct operators [11]. Therefore, 2 bit of classical memory per register qubit is always required at any measurement step of the cluster state MBQC. This means that $2 k T \ln 2$ of heat dissipation occurs at every step of the cluster state MBQC. In this way, the cluster state MBQC already achieves the minimum heat dissipation limit. Hence we also conclude that our general limit, $2 k T \ln 2$, is an achievable limit (i.e., not too underestimating limit.)

For the cluster state $\mathrm{MBQC}$, to derive the $2 k T \ln 2$ limit is somehow straightforward. However, we want to emphasise here that our limit $2 k T \ln 2$ for general MBQC given in the previous section does not make any assumption on the resource state, the way of measurements, and the way of classical processing. We have derived a general limit by using only the no-signaling principle. In a future someone might find a very complicated and drastically new MBQC far from the cluster state MBQC. (For example non-local many-body measurements might be allowed.) However, our minimum heat dissipation limit $2 k T \ln 2$ still holds for such a new MBQC as long as it satisfies the no-signaling principle.

Implication for blind quantum computation. - Blind quantum computation is a new secure cloud quantum computing protocol where a client (Alice), who does not have enough quantum technology, can delegate her quantum computation to a server (Bob), who has a fullfledged quantum computer, without leaking any information [39 53]. In blind quantum computing, the nosignaling requirement of the present result is replaced with the security requirement that server's state must be one-time padded. As is pointed out in Ref. 39], the client requires only a classical computer if she interacts with two servers. In this case, we have only to minimize client's classical technological requirement. Our result suggests that the client cannot be completely free from any technology; she has to possess at least 2 bit of classical memory per qubit in blind quantum computing.

Implication for classical technology requirement in $M B Q C$. - The requirement for classical technology in $\mathrm{MBQC}$ is a research subject which has not been fully studied. Although classical computation is cheap compared with quantum operations, detailed understanding of the classical part is important, because for example the latency of classical computation could contribute to the entire decoherence time of MBQC. The requirement for the power of the classical CPU was studied in Ref. [59]: classical XOR gate is sufficient for universal MBQC with cluster state, whereas a classical universal gate set is necessary if we use certain resource states (quantum computational tensor-network states [14]) in stead of the cluster state. Our result clarifies the minimum classical memory requirement, namely two bits per qubit, for MBQC.

Heat generation from other degrees of freedom.- As is already pointed out by Landauer himself [1], heat generation from Landauer's principle is not a practical limit, but a fundamental one: it is absurd to use Landauer's principle to estimate the overall heat generation in an iPhone, since there are many other factors which contribute to the entire heat generation, and these contributions are many orders of magnitude larger than that from Landauer's principle. This is also the case for our result. For example, measuring process could cause heat dissipation. If a qubit is encoded in a polarization of a single photon, or a two-level of an atom, a photon detection is necessary for the measurement. Usually, a photon detection generates a dissipative electric current, which generates heat of many magnitude larger than the Landauer limit. However, these contributions are implementation specific, and therefore beyond the scope of the present paper. (For example, the specific measurement model which uses photons by Brillouin [6] needs some energy for demon.) The purpose of the present paper is to derive a fundamental limit that is independent from any specific implementation, like the original motivation of Landauer's paper [1].

TM is supported by Program to Disseminate Tenure Tracking System by MEXT, Japan.

* Electronic address: morimae@gunma-u.ac.jp

[1] R. Landauer, Irreversibility and heat generation in the computing process, IBM J. Res. Dev. 5, 183 (1961).

[2] A. Bérut, A. Arakelyan, A. Petrosyan, S. Ciliberto, R. Dillenschneider, and E. Lutz, Experimental verification of Landauer's principle linking information and thermodyanmics. Nature 483, 187 (2012).

[3] L. Szilard, Z. Phys. 53, 840 (1929).

[4] C. H. Bennett, Logical reversibility of computation. IBM J. Res. Dev. 17, 525 (1987).

[5] C. H. Bennett and R. Landauer, The fundamental physical limits of computation. Sci. Am. 253, 48 (1985).

[6] L. Brillouin, Maxwell's demon cannot operate: information and entropy. I. J. Appl. Phys. 22, 334 (1951).

[7] K. Shizume, Heat generation required by information erasure. Phys. Rev. E 52, 3495 (1995).

[8] K. Maruyama, F. Nori, and V. Vedral, The physics of Maxwell's demon and information. Rev. Mod. Phys. 81, $1,(2009)$.

[9] C. H. Bennett, The thermodynamics of computation - a review. Int. J. Theor. Phys. 21, 905 (1982).

[10] T. Sagawa and M. Ueda, Minimal energy cost for thermodynamic information processing: measurement and infor- 
mation erasure. Phys. Rev. Lett. 102, 250602 (2009).

[11] R. Raussendorf and H. J. Briegel, A one-way quantum computer. Phys. Rev. Lett. 86, 5188 (2001).

[12] S. Popescu and D. Rohrlich, Quantum nonlocality as an axiom. Found. Phys. 24, 379 (1994).

[13] F. Verstraete and J. I. Cirac, Valence bond solids for quantum computation. Phys. Rev. A 70, 060302(R) (2004).

[14] D. Gross and J. Eisert, Novel schemes for measurementbased quantum computation. Phys. Rev. Lett. 98, 220503 (2007).

[15] G. K. Brennen and A. Miyake, Measurement-based quantum computer in the gapped ground state of a two-body Hamiltonian. Phys. Rev. Lett. 101, 010502 (2008).

[16] J. Cai, W. Dür, M. Van den Nest, A. Miyake, and H. J. Briegel, Quantum computation in correlation space and extremal entanglement. Phys. Rev. Lett. 103, 050503 (2009).

[17] A. Miyake, Quantum computation on the edge of a symmetry-protected topological order. Phys. Rev. Lett. 105, 040501 (2010).

[18] A. Miyake, Quantum computational capability of a twodimensional velence bond solid phase. Ann. Phys. 326, 1656 (2011).

[19] T. C. Wei, I. Affleck, and R. Raussendorf, The 2D AKLT state is a universal quantum computational resource. Phys. Rev. Lett. 106, 070501 (2011).

[20] J. Cai, A. Miyake, W. Dür, and H. J. Briegel, Universal quantum computer from magnet. Phys. Rev. A 82, 052309 (2010).

[21] Y. J. Chiu, X. Chen, and I. L. Chuang, Fermionic measurement-based quantum computation. Phys. Rev. A 87, 012305 (2013).

[22] T. Morimae, How to upload a physical state to the correlation space. Phys. Rev. A 83, 042337 (2011).

[23] T. Morimae, Quantum computational tensor network on string-net condensate. Phys. Rev. A 85, 062328 (2012).

[24] S. Bravyi and R. Raussendorf, On measurement-based quantum computation with the toric code states. Phys. Rev. A 76, 022304 (2007).

[25] M. Van den Nest, W. Dür, and H. J. Briegel, Classical spin models and the quantum stabilizer formalizm. Phys. Rev. Lett. 98, 117207 (2007).

[26] M. Van den Nest, W. Dür, and H. J. Briegel, Completeness of the classical 2D Ising model and universal quantum computation. Phys. Rev. Lett. 100, 110501 (2008).

[27] R. Raussendorf, J. Harrington, and K. Goyal, Topological fault-tolerance in cluster state quantum computation. New J. Phys. 9, 199 (2007).

[28] S. D. Barrett and T. M. Stace, Fault-tolerant quantum computation with very high threshold for loss errors. Phys. Rev. Lett. 105, 200502 (2010).

[29] K. Fujii and Y. Tokunaga, Fault-tolerant topological oneway quantum computation with probabilistic two-qubit gates. Phys. Rev. Lett. 105, 250503 (2010).

[30] Y. Li, S. D. Barrett, T. M. Stace, and S. C. Benjamin, Fully fault-tolerant quantum computation with non-deterministic gates. Phys. Rev. Lett. 105, 250502 (2010).

[31] Y. Li, D. E. Browne, L. C. Kwek, R. Raussendorf, and T. C. Wei, Topological quantum computation with spin-2 and 3/2 system. Phys. Rev. Lett. 107, 060501 (2011).

[32] K. Fujii and T. Morimae, Topologically protected measurement-based quantum computation on the ther- mal state of a nearest-neighbor two-body Hamiltonian with spin-3/2 particles. Phys. Rev. A 85, 010304(R) (2012).

[33] K. Fujii and T. Morimae, Computational power and correlation in quantum computational tensor network. Phys. Rev. A 85, 032338 (2012).

[34] D. Gross, S. T. Flammia, and J. Eisert, Most quantum states are too entangled to be useful as computational resources. Phys. Rev. Lett. 102, 190501 (2009).

[35] M. Bremner, C. Mora, and A. Winter, Are random pure states useful for quantum computation? Phys. Rev. Lett. 102, 190502 (2009).

[36] M. Van den Nest, W. Dür, G. Vidal, and H. J. Briegel, Classical simulation versus universality in measurement based quantum computation. Phys. Rev. A 75, 012337 (2007).

[37] M. Van den Nest, A. Miyake, W. Dür, and H. J. Briegel, Universal resource for measurement-based quantum computation. Phys. Rev. Lett. 97, 150504 (2006).

[38] T. Morimae, Strong entanglement causes low gate fidelity in inaccurate one-way quantum computation. Phys. Rev. A 81, 060307(R) (2010).

[39] A. Broadbent, J. Fitzsimons, and E. Kashefi, Universal blind quantum computation. Proc. of the 50th Annual IEEE Sympo. on Found. of Comput. Sci. 517 (2009).

[40] J. Fitzsimons and E. Kashefi, Unconditionally verifiable blind computation. arXiv:1203.5217.

[41] S. Barz, E. Kashefi, A. Broadbent, J. Fitzsimons, A. Zeilinger, and P. Walther, Demonstration of blind quantum computing. Science 335, 303 (2012).

[42] V. Dunjko, E. Kashefi, and A. Leverrier, Blind quantum computing with weak coherent pulses. Phys. Rev. Lett. 108, 200502 (2012).

[43] T. Morimae, V. Dunjko, and E. Kashefi, Ground state blind quantum computation on AKLT state. arXiv: 1009.3486

[44] T. Morimae and K. Fujii, Blind topological measurementbased quantum computation. Nature Comm. 3, 1036 (2012).

[45] T. Morimae, Continuous-variable blind quantum computation. Phys. Rev. Lett. 109, 230502 (2012).

[46] T. Morimae, No-signaling topological quantum computation in intelligent environment. arXiv:1208.1495.

[47] T. Morimae and K. Fujii, Blind quantum computation for Alice who does only measurements. Phys. Rev. A 87, 050301(R) (2013).

[48] T. Sueki, T. Koshiba, and T. Morimae, Ancilla-driven universal blind quantum computation. Phys. Rev. A 87, 060301(R) (2013).

[49] V. Dunjko, J. F. Fitzsimons, C. Portmann, and R. Renner, Composable security of delegated quantum computation. arXiv:1301.3662

[50] T. Morimae and T. Koshiba, Composable security of measuring-Alice blind quantum computation. arXiv:1306.2113

[51] T. Morimae and K. Fujii, Secure entanglement distillation for double-server blind quantum computation. arXiv:1302.3786 to be published in Phys. Rev. Lett.

[52] V. Giovannetti, L. Maccone, T. Morimae, and T. G. Rudolph, Efficient universal blind computation. arXiv:1306.2724

[53] A. Mantri, C. A. Pérez-Delgado, and J. F. Fitzsimons, Optimal blind quantum computation. arXiv:1306.3677

[54] J. Von Neumann, Mathematical foundations of quan- 
tum mechanics, (Princeton University Press, Princeton, 1955).

[55] A. Nayak and P. Sen, Invertible quantum operations and perfect encryption of quantum states. Quant. Inf. Comput. 7, 103 (2007).

[56] P. O. Boykin and V. Roychowdhury, Optimal encryption of quantum bits. Phys. Rev. A 67, 042317 (2003).

[57] A. Ambainis, M. Mosca, A. Tapp, and R. de Wolf, Private quantum channels. Proceedings of 41st Annual Symposium on Foundations of Computer Science (2000).

[58] Sagawa and Ueda [10 also derived another inequality $W_{\text {meas }}+W_{\text {eras }} \geq k T I_{Q C}$, where $W_{\text {meas }}$ is the work required for the measurement, $W_{\text {eras }}$ is the work required for the erasure of the measurement result in the mem- ory, and $I_{Q C}$ is what they call "QC-mutual information". It is defined as $I_{Q C} \equiv S(\rho)-\sum_{j} p_{j} S\left(\rho^{j}\right)$, where $\rho$ is the pre-measurement state of the system, and $\rho^{j}$ is the post-measurement state of the system obtained with the probability $p_{j}$. However, if $\rho$ and $\left\{\rho^{j}\right\}$ are all pure, like the many cases in $\mathrm{MBQC}$, the above inequality is not useful since $I_{Q C}$ is always 0 . (For example, consider the $Z$-basis measurement on the state $|+\rangle=|0\rangle+|1\rangle$. The measurement gets one bit of information, whereas $\left.I_{Q C}=S(|+\rangle)-\frac{1}{2} S(|0\rangle)-\frac{1}{2} S(|1\rangle)=0.\right)$

[59] J. Anders and D. E. Browne, Computational power of correlations. Phys. Rev. Lett. 102, 050502 (2009). 